\title{
COMBINING RESULTS FROM AUGMENTED DESIGNS OVER SITES
}

BU-1457-M

August, 2000

\section{Walter T. Federer \\ Biometrics \\ Cornell University}

Matthew Reynolds

Wheat Program, CIMMYT, Mexico

Jose Crossa

Biometrics and Statistics Unit, CIMMYT, Mexico

Keywords: inter-regression information, inter-gradient information, inter-variety information, row-column design.

\begin{abstract}
The class of augmented designs contains two kinds of treatments, standard or check and new of augmented. The latter are usually considered to be random effects while the check treatments are considered as fixed effects. New treatments are usually not replicated and the checks are replicated as points of reference. An augmented experiment design is a screening design. It is obtained by selecting any experiment design for the c check treatments and then enlarging the blocks or increasing the number of rows and/or columns to accommodate the $n$ new treatments. The new treatments are randomly distributed among the blocks or among the rows and columns. An augmented design has many advantages over systematic check arrangements and is useful for shortening the selection cycle over standard methods. Statistical procedures are available for recovering inter-block or inter-row-column and inter-variety information at each site. Procedures are presented herein for combining the results from single experiments over sites. Inter-site information may be recovered for random site effects. The method recommended for combining results is invariant to experiment design changes, variance heterogeneity, changes in checks from site to site, and different response models for each site. An experiment involving 120 new wheat genotypes and checks was conducted at three sites. It is used to illustrate the statistical procedure for combining results over sites. The new wheat genotypes exhibited a relatively large genotype by site interaction, indicating their site and environmental specificity.
\end{abstract}




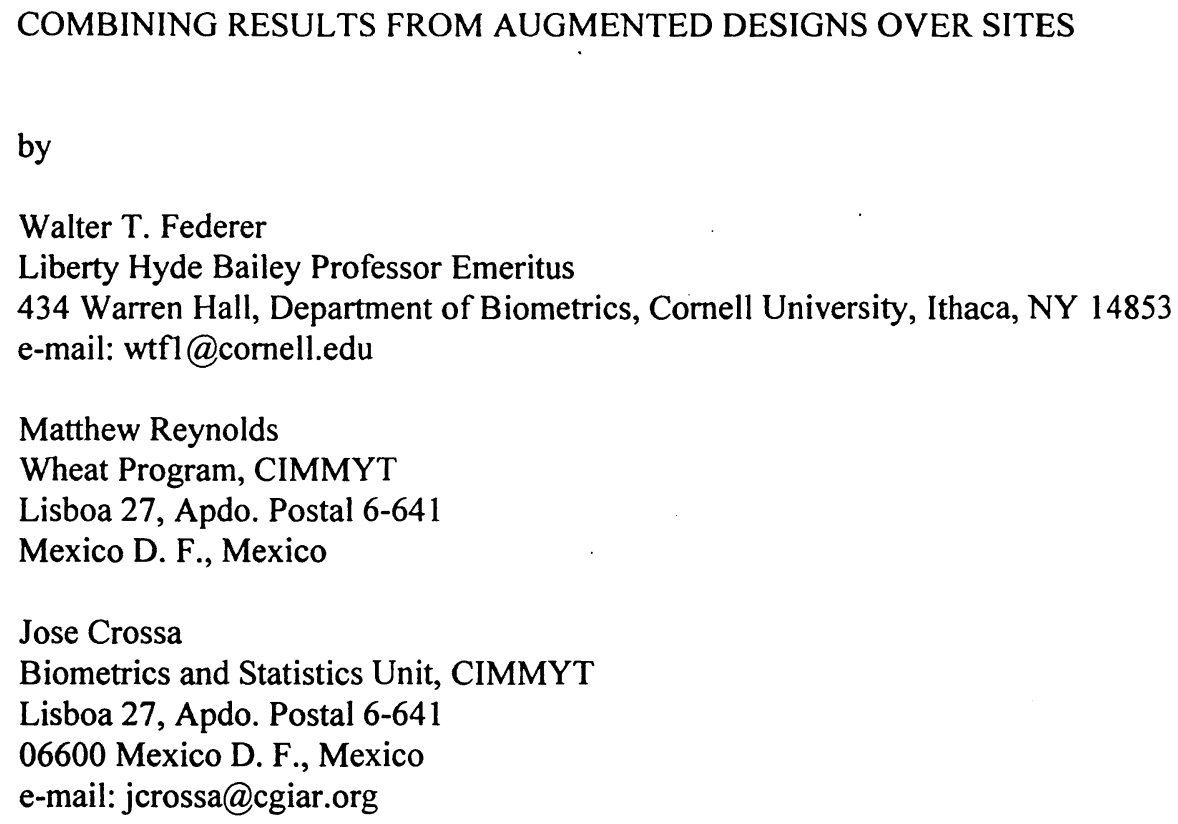

\section{ABSTRACT}

The class of augmented designs contains two kinds of treatments, standard or check and new or augmented. The latter are usually considered to be random effects while the check treatments are considered as fixed effects. New treatments are usually not replicated and the checks are replicated as points of reference. An augmented experiment design is a screening design. It is obtained by selecting any experiment design for the $c$ check treatments and then enlarging the blocks or increasing the number of rows and/or columns to accommodate the $\mathrm{n}$ new treatments. The new treatments are randomly distributed among the blocks or among the rows and columns. An augmented design has many advantages over systematic check arrangements and is useful for shortening the selection cycle over standard methods. Statistical procedures are available for recovering inter-block or inter-row-column and inter-variety information at each site. Procedures are presented herein for combining the results from single experiments over sites. Inter-site information may be recovered for random site effects. The method recommended for combining results is invariant to experiment design changes, variance heterogeneity, changes in checks from site to site, and different response models for each site. An experiment involving 120 new wheat genotypes and checks was conducted at three sites. It is used to illustrate the statistical procedure for combining results over sites. The new wheat genotypes exhibited a relatively large genotype by site interaction, indicating their site and environmental specificity.

Key words or phrases: inter-regression information, inter-gradient information, inter-variety information, row-column design, incomplete block design, polynomial regression, computer programs, genotype by environment interaction, variance component

\section{INTRODUCTION}

The class of augmented experiment designs (Federer, 1956, 1961, 1991; Federer et al., 1975a, 1975b) was introduced to replace the systematically spaced check arrangements for screening new genotypes in plant breeding research investigations. Usually material for the new treatments is limited, and it is necessary to include a new treatment only once. If the material is not limited but the number of new treatments is large, it is may be desirable to include new treatments only once. Federer $(1998)$ and Federer et al. $(1997,1998)$ have provided a statistical procedure for analyzing such experiments at a site. The analysis takes account of 
the random nature of the new treatments and of the blocking variables. This results in a more efficient analysis than if this information is ignored. Augmented designs have several advantages over the systematic check arrangement such as

(i) more than one check treatment may be included,

(ii) standard errors of differences between new treatments are available,

(iii) standard errors of differences between new and checks are available,

(iv) using the survivors of a previous screening stage as the checks is a device for testing the survivors at the same time that a new set of genotypes is being screened, and

(v) fewer cycles of selection are needed than for the standard method.

An augmented experiment design (AED) is useful for screening new treatments such as genotypes, insecticides, herbicides, drugs, etc. The number of new treatments, $n$, may be large, even in the hundreds and thousands. An AED is constructed by selecting an experiment design for the check treatments. The experiment design could be a randomized complete block (RCBD), an incomplete block (ICBD), a rowcolumn design, or some other design. If the selected design for check treatments is an ICBD, then the rb blocks (b incomplete blocks per complete block or replicate) are enlarged to accommodate $\mathrm{n} / \mathrm{rb}$ new treatments per incomplete block. If a row-column design is selected, the numbers of rows and columns are increased to include the $\mathrm{n}$ new treatments. If new treatments are included more than once, the analysis must take this into account. The augmented design analyses described herein are for one replicate of each new treatment. Responses may be obtained for all or for only a fraction of the new (augmented) treatments as only the replicated check treatment responses are used for obtaining solutions for blocking effects and an estimate of the error variance.

Using an appropriate response model for each site, analyses are presented for experiments at the different sites. The selected model needs to account for the spatial variation present in the experiment. Standard textbook models may be inappropriate, and a model needs to be selected from a class of plausible models (See, e.g., Federer, 1998). The standard procedure is to use a fixed effect analysis to select a response model and blocking parameters. Then, for the selected model, a mixed effect analysis of fixed checks and random blocking and new treatment effects is performed. The check means are adjusted for the information in the random blocking effects, i.e., recovery of inter-block, inter-regression (when regression is used as a blocking variable to explain trends in spatial variation), inter-row, or/and inter-column information. The new treatment means are adjusted by recovering inter-regression, inter-block, and/or inter-variety information. Recovery of random effect information results in adjusted means with smaller variances; it shrinks the size of fixed effect estimates by taking account of the random distribution of effects. Computer programs are available for both fixed and mixed effects analyses (Wolfinger et al., 1997). For each site, the most appropriate response model and best estimate of the new treatment means should be utilized. Utilizing the adjusted means for each site, it is demonstrated how to combine the results over sites. Two methods for combining results over sites are presented.

The main objective of the experiment used for an illustrative example in this paper, was to measure genetic diversity for physiological performance within a family of spring wheat lines derived from a cross between a heat tolerant and a heat sensitive parent (Reynolds et al., 1998). Quantitative traits such as those associated with heat tolerance may show considerable interaction with the environment. Therefore, the testing of new lines requires extensive evaluation in different locations (environments) to establish their genetic potential. Improved methods for identifying superior lines can result in significant savings. One approach is to reduce the number of replications used in a single field trial, assuming that performance can still be evaluated accurately. Augmented designs were developed for such situations.

\section{MATERIALS AND METHODS}

The example selected to illustrate the statistical procedures is a wheat variety trial conducted at three sites by the Physiology Unit of CIMMYT's Wheat Program. The experiment design at two of the sites was a 15 row by 12 column design. The treatment design was $c=2$ checks and $n=120$ new genotypes. The check treatments were replicated 30 times each and the new genotypes only once at each site. The two checks 
appeared twice in each of the 15 rows. The field layouts, grain weights, and other responses at the two sites are available from the authors. The particular design used is not connected, i.e., not all row, column, and treatment effects have solutions, the rank being two less than needed for connectedness. Polynomial regressions for trend in rows and in columns were used for the analysis. A check treatment occurred on every third diagonal, indicating that the rows and columns of the design plan were not randomized for the field layout. This non-randomized arrangement may bias the residual mean square upward. The size of the experimental unit or plot was $5 \mathrm{~m}$ by $1.2 \mathrm{~m}$. An appropriate randomization procedure for designs of the row-column type may be found in standard texts.

The experiment design at the third site was an incomplete block design of $v=120$ treatments (the new treatments at the above two sites) in incomplete blocks of size $k=8$. Check varieties were not included at this site. The 15 incomplete blocks in each of $r=2$ replicates were laid out adjacent to each other effectively making an eight column by 15 row lay-out in each replicate. The spatial layout of the experiment indicates that the design of the experiment should be considered as a resolvable 15 -row by 8 column design rather than as an incomplete block design.

At sites 1 and 2, polynomial functions of the rows and column effects were used to determine which functions of rows and columns describe the variation present in the experiment. Polynomial regressors use centered values of the powers of the independent variable. Hence, any of the regression coefficients may be large or small depending upon the nature of the spatial variation. The SAS/GLM procedure was used to obtain the analysis of variance (ANOVA). Polynomial functions for rows, $\mathrm{R}^{\mathrm{j}}$, and columns, $\mathrm{C}^{\mathrm{i}}$, were fitted, up to twelfth degree for rows $(j=1, \ldots, 12)$ and up to tenth degree $(i=1, \ldots, 10)$ for columns (See Federer and Wolfinger, 1998) as the rank of the normal equations was two less than needed for a row, column, treatment ANOVA. Fitting row effects is equivalent to using a fourteenth degree polynomial. Polynomial regression up to twelfth degree was used as the rank was two less than needed to estimate all row effects. Using the procedure described by Bozivich et al. (1956) and Federer et al. (1998), all terms in the polynomials with $\mathrm{F}$-values less than the $25 \%$ level were relegated to the residual category. The remaining regressors were treated as random blocking effects for explaining the spatial variation present in the experiment. This rule results in a slight change in the Type I error probability levels (Bozivich et al., 1956). Since the gradients in a field are not always in the same direction as the row-column layout, interactions of polynomial regressors such as row-linear by column-linear, row-linear by column-quadratic, etc. were fitted to account for this type of variation. The interactions $C^{i *} R^{j}$ fitted were from $i=1$ to 4 and $j=1$ to 4 . Again, the Bozivich et al. (1956) procedure was used to determine which of the 16 interactions should be pooled with the residual and which should be retained to account for the spatial variation present in the experimental area. This selection procedure provides a safeguard against over-parameterization. For the third site, differential linear gradients within the incomplete blocks accounted for the spatial variability present in the experiment.

The resulting fixed effect response models selected for the three sites were:

Site 1:

Yield= entry $\mathrm{Cl}$ C2 $\mathrm{C} 3 \mathrm{C} 4 \mathrm{C} 6 \mathrm{C} 8 \mathrm{R} 1 \mathrm{R} 2 \mathrm{R} 4 \mathrm{R} 8 \mathrm{R} 10 \mathrm{Cl}$ *R1 C2*R1 C3*R1

Site 2:

Yield = entry $\mathrm{Cl} 1 \mathrm{C} 4 \mathrm{C} 10 \mathrm{R} 2 \mathrm{Cl}^{*} \mathrm{R} 1 \mathrm{Cl}^{*} \mathrm{R} 3 \quad \mathrm{C} 2 * \mathrm{R} 2 \mathrm{C} 2 * \mathrm{R} 4 \quad \mathrm{C} 3 * \mathrm{R} 2 \quad \mathrm{C} 3 * \mathrm{R} 4 \mathrm{C} 4 * \mathrm{R} 3 \quad \mathrm{C} 4 * \mathrm{R} 4$

Site 3:

Yield $=$ replicate entry block(replicate) $\mathrm{Cl}$ *block(replicate)

$\mathrm{Ci}$ and $\mathrm{Rj}$ represent the orthogonal polynomial regression coefficients of degree $\mathrm{i}$ for columns and degree $\mathrm{j}$ for rows, respectively. The models are written in the form required by the SAS/GLM procedure. All elements in the above response models for sites 1 and 2 except for the check treatments are considered to be random effects. Since the 120 new genotypes are untested, they are considered to be random effects (See Federer, 1998). The goal of model selection is to find the response model that explains the spatial variation present in the experiment. Higher degree polynomials and interactions are required to account for non- 
uniform gradients as opposed to uniform ones. The ordering of regression degree is irrelevant; no explanation is needed beyond that it accounts for the spatial variation present in the experiment.

For site 3, only the 120 new treatments were included and it is still possible to consider the new treatment effects as random using the SAS/MIXED procedure. The following is the SAS code for the site 3 analysis:

PROC MIXED METHOD = REML; CLASS REP BLOCK ENTRY; MODEL YIELD = /SOLUTION; RANDOM REP BLOCK(REP) C1*BLOCK(REP) ENTRY;

Notice that no effects are listed in the MODEL statement since only fixed effects can be included here. With the SOLUTION option in the MODEL statement and ENTRY listed in the RANDOM statement, one needs to add the intercept value to the solution for the entry effect to obtain the mean (called REML or BLUP mean). In the SAS/MIXED procedure, one may use the default option, restricted maximum likelihood (REML), or one of the other options such as, e.g., analysis of variance (ANOVA) solutions for the variance components. This is done using the statement

\section{PARMS (A) (B) (C) /NOITER;}

where $\mathrm{X}$ in $(\mathrm{X})$ is the value for a variance component. "NOITER" denotes no iterations are to be used. This statement appears after the RANDOM statement. Also, the word NOPROFILE needs to be added in the PROC MIXED statement. This procedure is useful when the analyst wishes to use ANOVA instead of REML solutions for the variance components. ANOVA solutions are unbiased and do not depend upon the normality assumption.

Pertinent references for combining results from a series of experiments in a different context are Cochran (1939), Yates and Cochran (1938), Federer (1951), Cochran and Cox (1957), references on analysis of crop rotations, and references on meta-analysis. Combined analyses using means per site is simple and straightforward. As suggested by Cullis et al. (1996) and Frensham et al. (1997) for the case of replicated experiments, heterogeneity of within site error variance can be considered by using the reciprocal of the standard errors of the means. Crossa and Cornelius (1997) used this transformation in the multiplicative site regression model with the purpose of identifying subsets of sites without genotypic crossover genotype by environment interaction. Piepho (1999) suggested a weighted two-stage analysis across sites where, in the first stage, the treatment-site means and their associated error variances are obtained considering sites as fixed effects. The second stage considers a mixed model with sites and genotypes by site interaction as random effects and where weights of the reciprocal of the standard error of a mean are used.

Several procedures are available for combining results over sites. Some of these are

(i) a fixed effects analysis of rows, columns, sites, and entries,

(ii) a mixed model analysis of random effects sites, rows, columns, and new genotypes with checks as fixed effects and perhaps adding row by column interaction as random,

(iii) a fixed effects analysis on fixed effects means,

(iv) a mixed model analysis with site and new as random using the fixed effects means,

(v) a regression-site-new random effects analysis over sites with checks as fixed, or

(vi) a bootstrap analysis of the yields in Tables 1 and 2 or of those in Table 3.

The above methods are flawed in one respect or another with regard to the type of data considered here. Analysis (i) is easily accomplished using a software procedure like SAS/GLM but it does not make use of inter-regression and inter-variety information, and hence is inefficient. Analysis (ii) could be used using SAS/MIXED, say, as the REML solutions obtained depend upon the normal distribution and not on the over-parameterization or the connectedness of the design. Standard errors for over-parameterized models with REML will be inflated. Analysis (iii) is straightforward but does not make use of the random effects information. Analysis (iv) performs a fixed effect analysis at the individual sites and then considers sites 
and new entries as random effects using only the least squares fixed effect means for the new genotypes. Analysis ( $v$ ) requires the blocking (trend) effects to be the same at all sites, and hence is not usable here.

Several possibilities exist to test for genotype by site interaction. Analysis (i) could be used in some situations. Also, an average effective error of the means or solutions could be obtained and used as an error term to test for site by genotype interaction in analyses (iii), (iv), and (v). In many situations, a test for genotype by site interaction may be of minor interest. Instead, the plant breeder selects those entries having the highest yields over all sites and environments. It may be that selections will be made for specific environments. Since it is known that a genotype by environment interaction more than likely exists for the entries in this study, there is little interest in testing for it. For random sites, the genotype by site interaction mean square is the appropriate error mean square for testing genotype effects over sites. The new by site mean square has a different expectation than the check by site mean square. A comparison of new with check is the Behrens (also called the Behrens-Fisher) situation of unequal variances.

To overcome the flaws listed above, we shall use the following two methods for combining results from experiments over sites:

Method 1: Best estimates of new treatment means are obtained for each site. Then a two-way analysis of random site effects and fixed entry effects is performed. An estimate of the error variance over sites is obtained by computing the average effective error variance at each site and then averaging these over sites. This is a method suggested by Cochran and Cox (1957), Chapter 14. Their procedure is modified here to treat the new treatments as random effects.

Method 2: Best estimates of new treatment means are obtained for each site. These means are divided by their standard errors. These are called the standardized means and will have an expected error variance of unity. This means that estimates of variance components from a site by entry analysis will be ratios of the variance component to the error variance component.

These methods do not require the same experiment design at each site, do not require the same check treatments at each site, do not require the same response model at each site, and do not require homogeneity of error variances from site to site. These two methods are applied to the data from the three sites for means and for standardized means (smean). Also, since the two replicate means at site three were quite different, they were also treated as two sites. There was a heavy disease infection in one of the replicates. The unadjusted data from individual plots were used. A randomized complete block design variance was used to obtain the standard error for the data from the two replicates at site 3. Owing to the large residual (error) variance, this resulted in a lower weight being given to the data from site 3 . This is counter-balanced to some extent by using the replicates as two sites. The new treatments are considered as random effects in this case. Four sites were used for the third (means) and fourth (smeans) analyses of the data.

\section{RESULTS}

The 120 entry REML means obtained from the SAS/MIXED procedure for sites 1,2 , and 3 are presented in the second, third and fourth columns of Table 1 . The plot (eu) data from replicates 1 and 2 of the site 3 experiment are given in columns 5 and 6 of Table 1 as sites 4 and 5, respectively. Since none of the 120 entries were replicated in replicate 1 or 2 , the unadjusted plot data were used.

The means in Table 1 were divided by their standard errors to obtain the standardized means in Table 2. The standard error for site 1 was taken to be an average of the REML solution standard errors divided by the square root of two. The site 1 means were divided by 30.06 , site 2 means by 49.50 , and site 3 means by 154.00 to obtain the standardized means in columns 2,3, and 4 of Table 2. Since the data for sites denoted as 4 and 5 were unadjusted values, they were considered as coming from a randomized complete block design. The standard error for a single plot was 546.63. Dividing the data in columns 5 and 6 of Table 1 by 546.63 produced the standardized means in columns 5 and 6 of Table 2 . When data are standardized in this manner, the expected population error variance is unity, as in the unit normal distribution. This theoretical variance is the parameter and has infinite degrees of freedom. 
Sites were considered as random effects in computing the REML means over sites. The 120 entry means and standardized means for sites 1,2, and 3 and for sites 1,2, 4, and 5 are given in Table 3 . Each of the four sets of means averaged over sites has been ranked in descending order. The highest 15 means for sites 1,2 , and 3 were the following entries (Table 3)

$$
90,108,29,94,93,11,91,4,96,31,8,43,89,62,33
$$

For the smeans of sites 1,2, and 3 in column four, eight of the above 15 appeared in the top 15 smeans. For the means averaged over sites $1,2,4$, and 5 , ten of the top 15 were in agreement. For the standardized means over sites $1,2,4$, and 5 , entries $108,11,90,120,93,95,99,114,13,60,2$, and 53 appeared in the top 15 smeans for sites 1,2 , and 3 . The large error variance for the randomized complete block design analysis at site 3 resulted in small smeans and a shorter range of means at sites 4 and 5 . Thus, more weight is given to the data from sites 1 and 2. Similar comparisons may be made for the lowest yielding group of entries. For example, entries 44,69 , and 74 appeared at the bottom for means. The same entries appeared at the bottom or near the bottom for the other three methods of combining results.

Analyses of variance (ANOVAs) were obtained for the four sets of means and are presented in Table 4. The site or environment means squares are relatively large compared to the site by entry (genotype by environment) interaction. This is probably explained by the fact that the three sites differed in planting date as well as location. For the experiment at Tlaltizapan, site 1 was planted in November and site 2 in February and both were controlled for disease. For the third site, Obregon, the experiment was sown in February and had a heavy stem rust infection. The later the sowing date the greater the decrease in grain yield in these environments. Environmental factors influenced by the location and sowing date included temperature, photo-period, and soil physical and chemical properties. Yields generally decline at the higher temperatures, mainly because the growing cycle is truncated. All of the factors are likely to interact with genotype. Since such environmental differences exist, there is the question as to whether some real explainable effect is present and should be utilized in the interpretation of the results.

F-tests could be performed but their relevance in this context is in question. Instead some multiple comparisons procedure like sub-set selection appears more appropriate. If tables of Dunnett's comparison with a control were available for more than 20 entries (See Bechhofer and Dunnett, 1988), one could use this procedure. To illustrate, let us suppose that the tabled value is 3.00 for 120 entries with 238 degrees of freedom for the entry error variance and that a $90 \%$ coverage is desired. Then, for the entry with the highest mean over sites 1,2 , and 3 , number 90 , the interval is computed as

$$
1576-3.00[2(33,663) / 3]^{1 / 2}=1576-449.4=1126.6
$$

Thus all means lower than 1126.6 are considered to be significantly different from entry 90 and all those higher than 1126.6 are not. The site by entry mean square, 33,663, was used as the error term since the sites are considered to be a random sample of sites. Instead of the above, a multiple range test could be utilized for comparing means. For the means from sites 1, 2, and 3, a $95 \%$ studentized multiple range test is computed as

$$
\mathrm{q}_{\mathrm{v}=120, \mathrm{df}=238, \mathrm{a}=.05}(\text { standard error of a mean })=6.15(33,663 / 3)^{1 / 2}=651.5 .
$$

Owing to the large genotype by environment interaction, a study needs to be made of the results at each of the three sites. The ANOVAs for the three sites are given in Table 5. The F-values indicate large entry differences at each site. The fifteen top yielding entries at each of the three sites are

Site $1: 60,21,11,99,2,35,118,58,111,46,120,61,38,82,90$

Site $2: 18,103,37,72,85,65,26,11,79,94,110,15,25,20,95$

Site 3: $90,29,31,4,93,94,108,62,96,91,8,43,32,33,11$

The fifteen lowest yielding entries at each of the three sites are 
Site $1: 66,5,107,55,42,56,28,17,6,51,52,43,44,81,50$

Site 2: $29,69,36,83,31,107,73,19,117,35,74,118,56,34,32$

Site 3: 66, 10, 102, 18, 110,111, 49, 113, 109, 98, 44, 71, 69, 100, 74

There is considerable disagreement in the ranking at the three sites: e.g., genotype 90 ranked fifteenth in site 1 and first in site 3 . Entry 29 ranked second in site 3 and $106^{\text {th }}$ in site 2 . Entry 110 ranked eleventh in site 2 but $106^{\text {th }}$ in site 3 . Many of these genotypes appear to be site-specific for yield.

Variance component estimates for genotype by environment interaction as well as site are often desired. To do this, it is necessary to obtain an estimate of the pooled error mean square. One procedure is to average the error variances at the three sites as

$$
(3,449+6,088+41,604 /(r=2)) / 3=10,113 .
$$

(Note that there were $r=2$ replicates of the 120 entries at site 3.) This gives equal weight to each site. Another procedure is to weight the error variances by their degrees of freedom as follows

$$
(151,762+280,070+2,537,822 / 2) /(44+46+61)=11,263 .
$$

Owing to the relatively large spatial variations at site 3 , and to some extent at site 2 , there is some genotype by environment interaction in the residual mean square. The site by entry interaction has an expected value of error variance component plus site by entry variance component. The site by entry variance component for means at sites 1,2 , and 3 would be $33,663-11,263=22,400$. The ratio of the site by entry variance component to the error variance component would be $22,400 / 11,263=1.99$. Note that 10,113 may be used in place of 11,263 if equal site weights are desired.

For the ANOVA on smeans for sites 1, 2, and 3, the expected value of the site by entry mean square is $1+$ the site by entry variance component divided by the error variance component. Thus only a ratio of the two variance components can be obtained from the ANOVA on smeans. Ratios of variance components to the error variance component are used in measures of genetic advance (See, e.g., Sprague and Federer, 1951). For this example for smeans for sites 1,2 , and 3 , this ratio is $2.63-1=1.63$, or roughly equal to that obtained above.

\section{CONCLUSIONS}

Statistical difficulties encountered in combining results from experiments conducted at a number of sites may be overcome using either of the two methods described herein. Since the two methods used do not depend upon

(i) homogeneity of error variance,

(ii) using the same checks at every site,

(iii) having the same response model at every site, and/or

(iv) having the same experiment design at every site,

they are recommended for use by analysts when combining groups of experiments with the same entries at every site. If it is desired to give equal weight to each site, then Method 1 should be used. In order to weight responses in relation to their error variance at each site, it is recommended that standardized means, Method 2, be used. Site means with high error variances are given small weights. Method 2 has the property that it is unit free. For example, the grain weights may be measured in grams at one site and pounds at another site. Using standardized means, the need for conversion of measurements to the same scale is unnecessary. Standardized means are unit-free in the same manner as a correlation coefficient, a $t$ statistic, or an F statistic. Standardization in this form may be used to compare measurements on a wetweight basis with ones on a dry-weight basis, for example. 
A large genotype by environment interaction for yield of these 120 wheat genotypes was found. It should be noted that each variable may have its own response model at a given site as they may be affected by different spatial variation patterns during the course of the experiment. A response model should be selected for each characteristic measured in an experiment. For yield, the large difference in variation at each site would indicate that some genotype by environment interaction was occurring at some sites. For site 3 , a large difference in replicate means occurred. For site 2 , there was considerable variation in yields from one part of the row-column design to another. Since this represents a change in environment, an interaction is possible and suspected.

Apart from the savings on land and management costs, reducing the number of repetitions at a site and increasing the number of sites, improves the efficiency with which certain traits are evaluated by reducing the time it takes to characterize a family of lines. An example would be where trait evaluation is sensitive to changes in environmental conditions such as temperature and radiation intensity, as is the case of canopy temperature measurement (Reynolds et al., 1998).

\section{ACKNOWLEGEMENT}

Appreciation is expressed for the constructive comments made by the referees for several clarifying and edifying comments. A clearer and more readable exposition is the result. The enlightening contribution of one of the referees for input on the SAS/MIXED procedure is thankfully acknowledged.

\section{LITERATURE CITED}

Bechhofer, R. E. and C. W. Dunnett (1988). Percentage points of multivariate Student t distributions. In Selected Tables in Mathematical Statistics, Volume 11, American Mathematics Society, Providence, Rhode Island.

Bozivich, H., T. A. Bancroft, and H. O. Hartley (1956). Power of analysis of variance test procedures for certain incompletely specified models. I. Annals of Mathematical Statistics 27:1017-1043.

Cochran, W. G. (1939). Long-term agricultural experiments. J. Royal Statistical Soc., B 6:104-148.

Cochran W. G. and G. M. Cox (1957). Experimental Designs. John Wiley \& sons, Inc., New York.

Crossa, J. and P. L. Cornelius (1997). Site regression and shifted multiplicative model clustering of cultivar trial sites under heterogeneity of error variance. Crop Science 37:406-415.

Cullis, B. R., F. M. Thomson, J. A. Fisher, A. R. Gilmour, and R. Thompson (1996a). The analysis of the NSW wheat variety database: I. Modelling trial error variance. Theoretical and Applied Genetics 92:21-27.

Federer, W. T. (1951). Evaluation of variance components from a group of experiments with multiple classifications. Iowa Agric. Expt. Sta. Bulletin 380:241-310.

Federer, W. T. (1956). Augmented (or hoonuiaku) designs. Hawaiian Planters' Record LV(2):191-208.

Federer, W. T. (1961). Augmented designs with one-way elimination of heterogeneity. Biometrics 17:447473.

Federer, W. T. (1991). Statistics and Society. Marcel Dekker, Inc., New York, Section 7.11.

Federer, W. T. (1998). Recovery of interblock, intergradient, and intervariety information in incomplete block and lattice rectangle designed experiments. Biometrics 54:471-481. 
Federer, W. T., J. Crossa, and J. Franco (1998). New forms of spatial analyses with mixed model effects and exploratory model selection. BU-1406-M in the Technical Report Series of the Department of Biometrics, Cornell University, Ithaca, New York, April.

Federer, W. T., R. C. Nair, and D. Raghavarao (1975). Some augmented row-column designs. Biometrics 31:361-373.

Federer, W. T., E. A. Newton, and N. S. Altman (1997). Combining standard block analyses with spatial analyses under a random effects model. In Proceedings, Conference on Modelling Longitudinal and Spatially Correlated Data (Editors T. G. Gregoire et al.), Springer, New York, pp. 373-386.

Federer, W. T. and D. Raghavarao (1975). On augmented designs. Biometrics 31:29-35.

Federer, W. T. and R. D. Wolfinger (1998). SAS code for recovering intereffect information in experiments with incomplete block and lattice rectangle designs. Agronomy J. 90:545-551.

Frensham, A., B. Cullis, and A. Verbyla (1997). Genotype by environment variance heterogeneity in a twostage analysis. Biometrics 53:1373-1383.

Piepho, H-P. (1999). Stability analysis using the SAS system. Agronomy J. 91:154-160.

Reynolds, M. P., R. P. Singh, A. Ibrahim, O. A. A. Aggeb, A. Larque-Saavedra, and J. S. Quick (1998). Evaluating physiological traits to complement empirical selection for wheat in warm environments.

Euphytica 100:85-94.

Sprague, G. F. and W. T. Federer (1951). A comparison of variance components in corn yield trials: II Error, year $\times$ variety, location $\times$ variety, and variety components. Agronomy Journal 43:535-541.

Wolfinger, R. D., W. T. Federer, and O. Cordero-Brana (1997). Recovering information in augmented designs, using SAS PROC GLM and PROC MIXED. Agronomy J. 89:856-859.

Yates, F. and W. G. Cochran (1938). The analysis of groups of experiments. J. Agric. Sci. 28:556-580. 


\begin{tabular}{rllllll}
1 & \multicolumn{5}{c}{ lite } \\
2 & Table 1. REML & means by sites. & & \\
3 & & & & & \\
4 & & & & Site & & \\
5 & Entry1 & 1 & 2 & 3 & 4 & 5 \\
6 & 1 & 857 & 915 & 2071 & 2132 & 2693 \\
7 & 2 & 942 & 946 & 2394 & 2448 & 2331 \\
8 & 3 & 906 & 878 & 2414 & 2252 & 2717 \\
9 & 4 & 891 & 807 & 2735 & 3115 & 2520 \\
10 & 5 & 851 & 919 & 1896 & 1503 & 1233 \\
11 & 6 & 836 & 881 & 2372 & 3103 & 2540 \\
12 & 7 & 877 & 917 & 2308 & 2293 & 2332 \\
13 & 8 & 891 & 924 & 2579 & 2522 & 2496 \\
14 & 9 & 904 & 983 & 1852 & 1956 & 1265 \\
15 & 10 & 881 & 985 & 1756 & 2139 & 1205 \\
16 & 11 & 947 & 1036 & 2497 & 2472 & 2578 \\
17 & 12 & 920 & 1051 & 2247 & 2653 & 1658 \\
18 & 13 & 913 & 1031 & 2127 & 2599 & 1890 \\
19 & 14 & 896 & 861 & 1918 & 2191 & 1999 \\
20 & 15 & 883 & 1032 & 1836 & 1912 & 1134 \\
21 & 16 & 865 & 1032 & 1988 & 2311 & 1578 \\
22 & 17 & 839 & 1041 & 2254 & 2503 & 1624 \\
23 & 18 & 921 & 1128 & 1739 & 1867 & 1262 \\
24 & 19 & 875 & 842 & 2295 & 2108 & 2427 \\
25 & 20 & 882 & 1030 & 2207 & 2814 & 1612 \\
26 & 21 & 951 & 882 & 2118 & 2606 & 1800 \\
27 & 22 & 872 & 959 & 2363 & 3011 & 1639 \\
28 & 23 & 851 & 905 & 2375 & 2877 & 2506 \\
29 & 24 & 902 & 1021 & 1878 & 1832 & 1983 \\
30 & 25 & 887 & 1030 & 2116 & 2255 & 1889 \\
31 & 26 & 875 & 1070 & 2022 & 1932 & 2057 \\
32 & 27 & 883 & 938 & 2166 & 2237 & 2411 \\
33 & 28 & 846 & 854 & 1841 & 1863 & 2620 \\
34 & 29 & 863 & 845 & 2849 & 3053 & 3156 \\
35 & 30 & 886 & 925 & 2316 & 2349 & 1928 \\
36 & 31 & 871 & 801 & 2744 & 2988 & 2999 \\
37 & 32 & 896 & 728 & 2508 & 2920 & 2631 \\
38 & 33 & 920 & 873 & 2502 & 2694 & 2639 \\
39 & 34 & 868 & 697 & 2311 & 2760 & 2465 \\
40 & 35 & 937 & 800 & 2188 & 1790 & 2006 \\
41 & 36 & 921 & 811 & 1970 & 2435 & 1236 \\
42 & 37 & 909 & 1017 & 1871 & 1878 & 1432 \\
43 & 38 & 927 & 930 & 1898 & 2103 & 1099 \\
44 & 39 & 924 & 914 & 2071 & 2575 & 1429 \\
45 & 40 & 872 & 808 & 2158 & 2994 & 1785 \\
46 & 41 & 883 & 923 & 2016 & 2442 & 1948 \\
47 & 42 & 847 & 1029 & 2138 & 2070 & 1433 \\
48 & 43 & 808 & 965 & 2564 & 3718 & 2458 \\
49 & 44 & 808 & 824 & 1603 & 1670 & 895 \\
50 & 45 & 920 & 919 & 2132 & 2280 & 2266 \\
51 & 46 & 932 & 973 & 2071 & 2262 & 1905 \\
52 & 47 & 881 & 997 & 1854 & 1724 & 1919 \\
53 & 48 & 922 & 901 & 1778 & 1955 & 1880 \\
54 & 49 & 904 & 989 & 1707 & 1977 & 1099 \\
55 & 50 & 796 & 888 & 1841 & 1901 & 1068
\end{tabular}




$\begin{array}{rllllll}1 & 51 & 830 & 978 & 1930 & 2082 & 1746 \\ 2 & 52 & 813 & 939 & 1980 & 2231 & 1800 \\ 3 & 53 & 913 & 961 & 2351 & 2028 & 3028 \\ 4 & 54 & 878 & 957 & 2378 & 3246 & 2424 \\ 5 & 55 & 847 & 959 & 1847 & 2074 & 1591 \\ 6 & 56 & 846 & 717 & 2195 & 2386 & 2920 \\ 7 & 57 & 902 & 958 & 1894 & 2323 & 1679 \\ 8 & 58 & 937 & 916 & 2320 & 3009 & 1621 \\ 9 & 59 & 910 & 953 & 2116 & 2437 & 1744 \\ 10 & 60 & 974 & 1011 & 1997 & 1669 & 1843 \\ 11 & 61 & 931 & 855 & 2139 & 2498 & 2093 \\ 12 & 62 & 860 & 825 & 2637 & 3433 & 2194 \\ 13 & 63 & 879 & 1006 & 1976 & 2265 & 2058 \\ 14 & 64 & 872 & 881 & 1992 & 2380 & 1192 \\ 15 & 65 & 858 & 1019 & 1778 & 1456 & 1572 \\ 16 & 66 & 852 & 856 & 1774 & 1668 & 1126 \\ 17 & 67 & 908 & 953 & 2000 & 2552 & 1189 \\ 18 & 68 & 908 & 905 & 2188 & 2555 & 1527 \\ 19 & 69 & 889 & 859 & 1562 & 1386 & 1115 \\ 20 & 70 & 860 & 895 & 1946 & 2508 & 1271 \\ 21 & 71 & 853 & 970 & 1590 & 1892 & 1174 \\ 22 & 72 & 907 & 1017 & 1834 & 1976 & 1545 \\ 23 & 73 & 859 & 785 & 2039 & 2151 & 1909 \\ 24 & 74 & 866 & 688 & 1509 & 1640 & 1344 \\ 25 & 75 & 880 & 779 & 1995 & 2296 & 1844 \\ 26 & 76 & 899 & 975 & 1852 & 1903 & 1417 \\ 27 & 77 & 876 & 915 & 2073 & 2037 & 1523 \\ 28 & 78 & 877 & 962 & 2125 & 2243 & 1819 \\ 29 & 79 & 863 & 1026 & 2113 & 2651 & 1496 \\ 30 & 80 & 859 & 974 & 1967 & 2337 & 1744 \\ 31 & 81 & 802 & 978 & 2218 & 2122 & 1934 \\ 32 & 82 & 927 & 959 & 1981 & 2268 & 2076 \\ 33 & 83 & 896 & 800 & 1919 & 2139 & 2571 \\ 34 & 84 & 909 & 947 & 1891 & 1849 & 1049 \\ 35 & 85 & 899 & 1033 & 1949 & 1762 & 1866 \\ 36 & 86 & 907 & 987 & 1921 & 1771 & 1885 \\ 37 & 87 & 887 & 988 & 2285 & 2384 & 2359 \\ 38 & 88 & 886 & 919 & 1736 & 1938 & 1488 \\ 39 & 89 & 894 & 936 & 2493 & 2426 & 2051 \\ 40 & 90 & 926 & 901 & 2901 & 3158 & 3301 \\ 41 & 91 & 921 & 937 & 2596 & 2443 & 2232 \\ 42 & 92 & 918 & 992 & 2106 & 2718 & 1283 \\ 43 & 93 & 903 & 986 & 2663 & 2722 & 2670 \\ 44 & 94 & 872 & 1025 & 2656 & 2427 & 2297 \\ 45 & 95 & 910 & 1061 & 2301 & 1868 & 2526 \\ 46 & 96 & 880 & 948 & 2602 & 2569 & 2763 \\ 47 & 97 & 873 & 971 & 1791 & 2390 & 744 \\ 48 & 98 & 902 & 952 & 1612 & 1817 & 799 \\ 49 & 99 & 944 & 994 & 2117 & 2661 & 1712 \\ 50 & 100 & 876 & 956 & 1553 & 1999 & 1417 \\ 51 & 101 & 919 & 977 & 1955 & 2320 & 1558 \\ 52 & 102 & 891 & 1075 & 1745 & 2220 & 1615 \\ 53 & 103 & 876 & 1099 & 2014 & 2372 & 1447 \\ 54 & 104 & 867 & 985 & 1963 & 2761 & 1863 \\ 55 & 105 & 869 & 922 & 2283 & 2771 & 2314\end{array}$




$\begin{array}{rllllll}1 & 106 & 893 & 963 & 2396 & 2393 & 2260 \\ 2 & 107 & 848 & 853 & 1789 & 2302 & 1268 \\ 3 & 108 & 921 & 1016 & 2643 & 2578 & 3504 \\ 4 & 109 & 912 & 961 & 1613 & 2158 & 1558 \\ 5 & 110 & 891 & 1038 & 1724 & 1992 & 1021 \\ 6 & 111 & 934 & 922 & 1711 & 1797 & 938 \\ 7 & 112 & 857 & 971 & 1832 & 1688 & 1373 \\ 8 & 113 & 869 & 919 & 1628 & 2127 & 1525 \\ 9 & 114 & 906 & 989 & 2268 & 2506 & 2619 \\ 10 & 115 & 873 & 960 & 2287 & 2384 & 2592 \\ 11 & 116 & 890 & 922 & 2068 & 1938 & 1962 \\ 12 & 117 & 890 & 828 & 2052 & 2730 & 2123 \\ 13 & 118 & 937 & 762 & 2124 & 2416 & 1724 \\ 14 & 119 & 896 & 975 & 2005 & 2198 & 1370 \\ 15 & 120 & 932 & 988 & 2318 & 2687 & 2517 \\ 16 & & & & & & \end{array}$

Table 2. REML means divided by site means standard deviation.

30

$$
31
$$

$$
32
$$

$$
33
$$

$$
34
$$

34
35
36$$
\begin{aligned}
& 35 \\
& 36 \\
& 37
\end{aligned}
$$

37 37
38
39}

38
39
40

40

$$
\begin{aligned}
& 42 \\
& 43
\end{aligned}
$$

$$
\begin{aligned}
& 43 \\
& 44 \\
& 45
\end{aligned}
$$

44
45
46

$$
\begin{aligned}
& 46 \\
& 47 \\
& 48
\end{aligned}
$$

$$
30
$$$$
5
$$

5

$$
52
$$

53
54

\begin{tabular}{llllll} 
Entry & 1 & \multicolumn{5}{l}{ Site } & 3 & 4 & 5 \\
\hline 1 & 28.50 & 18.49 & 13.45 & 3.90 & 4.93 \\
2 & 31.34 & 19.11 & 15.55 & 4.48 & 4.26 \\
3 & 30.13 & 17.74 & 15.68 & 4.12 & 4.97 \\
4 & 29.65 & 16.30 & 17.76 & 5.70 & 4.61 \\
5 & 28.32 & 18.57 & 12.31 & 2.75 & 2.26 \\
6 & 27.80 & 17.80 & 15.40 & 5.68 & 4.65 \\
7 & 29.19 & 18.53 & 14.99 & 4.19 & 4.27 \\
8 & 29.63 & 18.66 & 16.75 & 4.61 & 4.57 \\
9 & 30.06 & 19.85 & 12.03 & 3.58 & 2.31 \\
10 & 29.30 & 19.89 & 11.40 & 3.91 & 2.20 \\
11 & 31.49 & 20.93 & 16.21 & 4.52 & 4.72 \\
12 & 30.59 & 21.24 & 14.59 & 4.85 & 3.03 \\
13 & 30.37 & 20.83 & 13.81 & 4.75 & 3.46 \\
14 & 29.81 & 17.39 & 12.45 & 4.01 & 3.66 \\
15 & 29.38 & 20.84 & 11.92 & 3.50 & 2.07 \\
16 & 28.76 & 20.85 & 12.91 & 4.23 & 2.89 \\
17 & 27.90 & 21.02 & 14.64 & 4.58 & 2.97 \\
18 & 30.65 & 22.78 & 11.29 & 3.42 & 2.31 \\
19 & 29.10 & 17.00 & 14.90 & 3.86 & 4.44 \\
20 & 29.35 & 20.82 & 14.33 & 5.15 & 2.95 \\
21 & 31.62 & 17.82 & 13.76 & 4.77 & 3.29 \\
22 & 29.01 & 19.38 & 15.34 & 5.51 & 3.00 \\
23 & 28.31 & 18.28 & 15.42 & 5.26 & 4.58 \\
24 & 30.01 & 20.62 & 12.19 & 3.35 & 3.63 \\
25 & 29.52 & 20.81 & 13.74 & 4.13 & 3.46 \\
26 & 29.09 & 21.61 & 13.13 & 3.53 & 3.76 \\
27 & 29.36 & 18.95 & 14.06 & 4.09 & 4.41 \\
28 & 28.15 & 17.25 & 11.95 & 3.41 & 4.79 \\
29 & 28.70 & 17.06 & 18.50 & 5.59 & 5.77 \\
30 & 29.47 & 18.69 & 15.04 & 4.30 & 3.53 \\
31 & 28.98 & 16.19 & 17.82 & 5.47 & 5.49 \\
32 & 29.81 & 14.70 & 16.29 & 5.34 & 4.81 \\
33 & 30.59 & 17.64 & 16.25 & 4.93 & 4.83 \\
34 & 28.89 & 14.07 & 15.003 & 5.05 & 4.51
\end{tabular}




\begin{tabular}{|c|c|c|c|c|c|c|}
\hline 1 & 35 & 31.18 & 16.16 & 14.21 & 3.27 & 3.67 \\
\hline 2 & 36 & 30.63 & 16.39 & 12.79 & 4.45 & 2.26 \\
\hline 3 & 37 & 30.24 & 20.55 & 12.15 & 3.44 & 2.62 \\
\hline 4 & 38 & 30.84 & 18.78 & 12.33 & 3.85 & 2.01 \\
\hline 5 & 39 & 30.73 & 18.46 & 13.45 & 4.71 & 2.61 \\
\hline 6 & 40 & 29.01 & 16.32 & 14.01 & 5.48 & 3.27 \\
\hline 7 & 41 & 29.38 & 18.65 & 13.09 & 4.47 & 3.56 \\
\hline 8 & 42 & 28.18 & 20.79 & 13.88 & 3.79 & 2.62 \\
\hline 9 & 43 & 26.87 & 19.49 & 16.65 & 6.80 & 4.50 \\
\hline 10 & 44 & 26.87 & 16.65 & 10.41 & 3.06 & 1.64 \\
\hline 11 & 45 & 30.59 & 18.56 & 13.84 & 4.17 & 4.15 \\
\hline 12 & 46 & 31.01 & 19.66 & 13.44 & 4.14 & 3.48 \\
\hline 13 & 47 & 29.32 & 20.15 & 12.04 & 3.15 & 3.51 \\
\hline 14 & 48 & 30.67 & 18.19 & 11.55 & 3.58 & 3.44 \\
\hline 15 & 49 & 30.06 & 19.99 & 11.08 & 3.62 & 2.01 \\
\hline 16 & 50 & 26.49 & 17.94 & 11.96 & 3.48 & 1.95 \\
\hline 17 & 51 & 27.61 & 19.75 & 12.53 & 3.81 & 3.19 \\
\hline 18 & 52 & 27.04 & 18.97 & 12.86 & 4.08 & 3.29 \\
\hline 19 & 53 & 30.36 & 19.42 & 15.26 & 3.71 & 5.54 \\
\hline 20 & 54 & 29.20 & 19.34 & 15.44 & 5.94 & 4.43 \\
\hline 21 & 55 & 28.18 & 19.38 & 11.99 & 3.79 & 2.91 \\
\hline 22 & 56 & 28.16 & 14.49 & 14.25 & 4.36 & 5.34 \\
\hline 23 & 57 & 30.00 & 19.34 & 12.30 & 4.25 & 3.07 \\
\hline 24 & 58 & 31.16 & 18.50 & 15.07 & 5.50 & 2.97 \\
\hline 25 & 59 & 30.27 & 19.24 & 13.74 & 4.46 & 3.19 \\
\hline 26 & 60 & 32.41 & 20.42 & 12.97 & 3.05 & 3.37 \\
\hline 27 & 61 & 30.96 & 17.26 & 13.89 & 4.57 & 3.83 \\
\hline 28 & 62 & 28.62 & 16.67 & 17.12 & 6.28 & 4.01 \\
\hline 29 & 63 & 29.26 & 20.33 & 12.83 & 4.14 & 3.76 \\
\hline 30 & 64 & 29.02 & 17.80 & 12.94 & 4.35 & 2.18 \\
\hline 31 & 65 & 28.53 & 20.59 & 11.55 & 2.66 & 2.88 \\
\hline 32 & 66 & 28.33 & 17.30 & 11.52 & 3.05 & 2.06 \\
\hline 33 & 67 & 30.19 & 19.26 & 12.99 & 4.67 & 2.18 \\
\hline 34 & 68 & 30.21 & 18.27 & 14.21 & 4.67 & 2.79 \\
\hline 35 & 69 & & 17. & 10 & 2.54 & 2.04 \\
\hline 36 & 70 & 28.61 & 18 & 12.64 & 4.59 & 2.33 \\
\hline 37 & 71 & 28.39 & 19.59 & 10.32 & 3.46 & 2.15 \\
\hline 38 & 72 & 30.17 & 20.55 & 11.91 & 3.61 & 2.83 \\
\hline 39 & 73 & 28.57 & 15.85 & 13.24 & 3.94 & 3.49 \\
\hline 40 & 74 & 28.82 & 13.91 & 9.80 & 3.00 & 2.46 \\
\hline 41 & 75 & 29.27 & 15.74 & 12.95 & 4.20 & 3.37 \\
\hline 42 & 76 & 29.90 & 19.70 & 12.02 & 3.48 & 2.59 \\
\hline 43 & 77 & 29.16 & 18.49 & 13.46 & 3.73 & 2.79 \\
\hline 44 & 78 & 29.16 & 19.43 & 13.80 & 4.10 & 3.33 \\
\hline 45 & 79 & 28.72 & 20.73 & 13.72 & 4.85 & 2.74 \\
\hline 46 & 80 & 28.59 & 19.69 & 12.77 & 4.28 & 3.19 \\
\hline 47 & 81 & 26.68 & 19.76 & 14.40 & 3.88 & 3.54 \\
\hline 48 & 82 & 30.83 & 19.38 & 12.86 & 4.15 & 3.80 \\
\hline 49 & 83 & 29.81 & 16.15 & 12.46 & 3.91 & 4.70 \\
\hline 50 & 84 & 30.23 & 19.13 & 12.28 & 3.38 & 1.92 \\
\hline 51 & 85 & 29.89 & 20.87 & 12.65 & 3.22 & 3.41 \\
\hline 52 & 86 & 30.18 & 19.93 & 12.47 & 3.24 & 3.45 \\
\hline 53 & 87 & 29.52 & 19.96 & 14.84 & 4.36 & 4.32 \\
\hline 54 & 88 & 29.47 & 18.57 & 11.28 & 3.55 & 2.72 \\
\hline 55 & 89 & 29.75 & 18.91 & 16.19 & 4.44 & 3.75 \\
\hline
\end{tabular}




$\begin{array}{rllllll}1 & 90 & 30.80 & 18.20 & 18.84 & 5.78 & 6.04 \\ 2 & 91 & 30.63 & 18.92 & 16.86 & 4.47 & 4.08 \\ 3 & 92 & 30.53 & 20.04 & 13.67 & 4.97 & 2.35 \\ 4 & 93 & 30.05 & 19.91 & 17.29 & 4.98 & 4.88 \\ 5 & 94 & 29.00 & 20.70 & 17.25 & 4.44 & 4.20 \\ 6 & 95 & 30.28 & 21.43 & 14.94 & 3.42 & 4.62 \\ 7 & 96 & 29.27 & 19.15 & 16.90 & 4.70 & 5.05 \\ 8 & 97 & 29.03 & 19.61 & 11.63 & 4.37 & 1.36 \\ 9 & 98 & 30.00 & 19.23 & 10.47 & 3.32 & 1.46 \\ 10 & 99 & 31.42 & 20.08 & 13.75 & 4.87 & 3.13 \\ 11 & 100 & 29.14 & 19.32 & 10.08 & 3.66 & 2.59 \\ 12 & 101 & 30.58 & 19.73 & 12.69 & 4.24 & 2.85 \\ 13 & 102 & 29.65 & 21.71 & 11.33 & 4.06 & 2.95 \\ 14 & 103 & 29.14 & 22.19 & 13.08 & 4.34 & 2.65 \\ 15 & 104 & 28.84 & 19.89 & 12.74 & 5.05 & 3.41 \\ 16 & 105 & 28.90 & 18.64 & 14.82 & 5.07 & 4.23 \\ 17 & 106 & 29.71 & 19.46 & 15.56 & 4.38 & 4.13 \\ 18 & 107 & 28.20 & 17.23 & 11.62 & 4.21 & 2.32 \\ 19 & 108 & 30.65 & 20.53 & 17.16 & 4.72 & 6.41 \\ 20 & 109 & 30.34 & 19.42 & 10.47 & 3.95 & 2.85 \\ 21 & 110 & 29.65 & 20.98 & 11.19 & 3.64 & 1.87 \\ 22 & 111 & 31.07 & 18.62 & 11.11 & 3.29 & 1.72 \\ 23 & 112 & 28.51 & 19.62 & 11.89 & 3.09 & 2.51 \\ 24 & 113 & 28.92 & 18.56 & 10.57 & 3.89 & 2.79 \\ 25 & 114 & 30.15 & 19.97 & 14.73 & 4.58 & 4.79 \\ 26 & 115 & 29.04 & 19.40 & 14.85 & 4.36 & 4.74 \\ 27 & 116 & 29.61 & 18.63 & 13.43 & 3.55 & 3.59 \\ 28 & 117 & 29.60 & 16.74 & 13.33 & 4.99 & 3.88 \\ 29 & 118 & 31.17 & 15.39 & 13.80 & 4.42 & 3.15 \\ 30 & 119 & 29.81 & 19.70 & 13.02 & 4.02 & 2.51 \\ 31 & 120 & 31.00 & 19.96 & 15.05 & 4.92 & 4.60 \\ 32 & & & & & & \end{array}$

32

33

34 35 36 37

38

39

$\begin{array}{lllll}40 & 108 & 1526 & 108 & 22.78\end{array}$

$\begin{array}{lllll}41 & 29 & 1519 & 90 & 22.61\end{array}$

$\begin{array}{lllll}42 & 94 & 1518 & 93 & 22.42\end{array}$

$\begin{array}{lllll}43 & 93 & 1517 & 94 & 22.32\end{array}$

$\begin{array}{lllll}44 & 11 & 1493 & 95 & 22.22\end{array}$

$\begin{array}{lllll}45 & 91 & 1484 & 12 & 22.14\end{array}$

$\begin{array}{lllll}46 & 4 & 1478 & 91 & 22.14\end{array}$

$\begin{array}{lllll}47 & 96 & 1477 & 120 & 22.00\end{array}$

$\begin{array}{lllll}48 & 31 & 1472 & 2 & 22.00\end{array}$

$\begin{array}{lllll}49 & 8 & 1465 & 60 & 21.93\end{array}$

$\begin{array}{lllll}50 & 43 & 1446 & 96 & 21.77\end{array}$

$\begin{array}{lllll}51 & 89 & 1441 & 99 & 21.75\end{array}$

$\begin{array}{lllll}52 & 62 & 1441 & 53 & 21.68\end{array}$

$\begin{array}{lllll}53 & 33 & 1432 & 8 & 21.68\end{array}$

$\begin{array}{lllll}54 & 2 & 1427 & 13 & 21.67\end{array}$

$\begin{array}{lllll}55 & 95 & 1424 & 89 & 21.62\end{array}$

Sites $1,2,4,5$

$\begin{array}{llll}\text { Entry } & \text { mean } & \text { Entry } & \text { smean } \\ 90 & 2072 & 108 & 15.58\end{array}$

$\begin{array}{llll}108 & 2005 & 11 & 15.42\end{array}$

$\begin{array}{llll}43 & 1987 & 90 & 15.21\end{array}$

$\begin{array}{llll}29 & 1979 & 120 & 15.12\end{array}$

$\begin{array}{llll}31 & 1915 & 93 & 14.96\end{array}$

$\begin{array}{llll}54 & 1876 & 95 & 14.94\end{array}$

$\begin{array}{llll}6 & 1840 & 12 & 14.93\end{array}$

$\begin{array}{llll}4 & 1833 & 99 & 14.88\end{array}$

$\begin{array}{llll}62 & 1828 & 114 & 14.87\end{array}$

$\begin{array}{llll}62 & 1828 & 114 & 14.87 \\ 93 & 1820 & 13 & 14.85\end{array}$

$\begin{array}{llll}32 & 1794 & 60 & 14.81\end{array}$

$\begin{array}{llll}96 & 1790 & 2 & 14.80\end{array}$

$\begin{array}{llll}23 & 1785 & 18 & 14.79\end{array}$

$\begin{array}{llll}33 & 1782 & 53 & 14.76\end{array}$

$\begin{array}{llll}120 & 1781 & 54 & 14.73\end{array}$

$\begin{array}{llll}11 & 1758 & 102 & 14.59\end{array}$

$\begin{array}{llll}114 & 1755 & 94 & 14.59\end{array}$ 


\begin{tabular}{|c|c|c|c|c|c|c|c|c|}
\hline 1 & 106 & 1417 & 114 & 21.62 & 53 & 1733 & 103 & 14.58 \\
\hline 2 & 120 & 1413 & 106 & 21.58 & 105 & 1719 & 46 & 14.57 \\
\hline 3 & 53 & 1408 & 58 & 21.58 & 56 & 1717 & 20 & 14.57 \\
\hline 4 & 12 & 1406 & 18 & 21.57 & 8 & 1708 & 96 & 14.54 \\
\hline 5 & 54 & 1404 & 20 & 21.50 & 115 & 1702 & 87 & 14.54 \\
\hline 6 & 3 & 1399 & 33 & 21.49 & 34 & 1698 & 82 & 14.54 \\
\hline 7 & 22 & 1398 & 103 & 21.47 & 3 & 1688 & 58 & 14.53 \\
\hline 8 & 58 & 1391 & 87 & 21.44 & 2 & 1667 & 91 & 14.53 \\
\hline 9 & 114 & 1388 & 29 & 21.42 & 94 & 1655 & 26 & 14.50 \\
\hline 10 & 87 & 1387 & 92 & 21.41 & 87 & 1655 & 33 & 14.50 \\
\hline 11 & 17 & 1378 & 46 & 21.37 & 1 & 1650 & 25 & 14.48 \\
\hline 12 & 32 & 1377 & 25 & 21.36 & 117 & 1643 & 92 & 14.47 \\
\hline 13 & 23 & 1377 & 54 & 21.33 & 91 & 1633 & 106 & 14.42 \\
\hline 14 & 30 & 1376 & 26 & 21.28 & 106 & 1627 & 43 & 14.42 \\
\hline 15 & 115 & 1373 & 22 & 21.24 & 58 & 1621 & 24 & 14.40 \\
\hline 16 & 20 & 1373 & 4 & 21.24 & 22 & 1620 & 115 & 14.39 \\
\hline 17 & 7 & 1367 & 17 & 21.19 & 104 & 1619 & 21 & 14.38 \\
\hline 18 & 6 & 1363 & 3 & 21.18 & 27 & 1617 & 63 & 14.37 \\
\hline 19 & 105 & 1358 & 85 & 21.14 & 40 & 1615 & 8 & 14.37 \\
\hline 20 & 13 & 1357 & 115 & 21.10 & 13 & 1608 & 45 & 14.37 \\
\hline 21 & 99 & 1352 & 59 & 21.08 & 7 & 1605 & 101 & 14.35 \\
\hline 22 & 25 & 1344 & 21 & 21.07 & 83 & 1602 & 85 & 14.35 \\
\hline 23 & 92 & 1339 & 30 & 21.07 & 45 & 1596 & 104 & 14.30 \\
\hline 24 & 42 & 1338 & 79 & 21.06 & 61 & 1594 & 59 & 14.29 \\
\hline 25 & 19 & 1337 & 82 & 21.02 & 95 & 1591 & 72 & 14.29 \\
\hline 26 & 79 & 1334 & 43 & 21.00 & 20 & 1585 & 29 & 14.28 \\
\hline 27 & 68 & 1334 & 101 & 21.00 & 99 & 1578 & 79 & 14.26 \\
\hline 28 & 81 & 1333 & 31 & 21.00 & 89 & 1577 & 3 & 14.24 \\
\hline 29 & 103 & 1330 & 45 & 21.00 & 12 & 1571 & 22 & 14.23 \\
\hline 30 & 27 & 1329 & 37 & 20.98 & 19 & 1563 & 37 & 14.21 \\
\hline 31 & 60 & 1328 & 42 & 20.95 & 21 & 1560 & 89 & 14.21 \\
\hline 32 & 59 & 1326 & 24 & 20.94 & 82 & 1558 & 105 & 14.21 \\
\hline 33 & 46 & 1325 & 7 & 20.90 & 63 & 1552 & 27 & 14.20 \\
\hline 34 & 45 & 1324 & 68 & 20.90 & 41 & 1549 & 86 & 14.20 \\
\hline 35 & 26 & 1322 & 102 & 20.90 & 28 & 1546 & 16 & 14.18 \\
\hline 36 & 78 & 1321 & 39 & 20.88 & 30 & 1522 & 57 & 14.17 \\
\hline 37 & 21 & 1317 & 72 & 20.88 & 46 & 1518 & 61 & 14.16 \\
\hline 38 & 35 & 1309 & 86 & 20.86 & 25 & 1515 & 109 & 14.14 \\
\hline 39 & 61 & 1308 & 119 & 20.84 & 59 & 1511 & 39 & 14.13 \\
\hline 40 & 39 & 1303 & 16 & 20.84 & 79 & 1509 & 17 & 14.12 \\
\hline 41 & 16 & 1295 & 67 & 20.81 & 17 & 1502 & 23 & 14.11 \\
\hline 42 & 85 & 1294 & 63 & 20.81 & 14 & 1487 & 67 & 14.08 \\
\hline 43 & 116 & 1293 & 62 & 20.80 & 26 & 1484 & 4 & 14.07 \\
\hline 44 & 34 & 1292 & 78 & 20.80 & 80 & 1479 & 7 & 14.05 \\
\hline 45 & 119 & 1292 & 27 & 20.79 & 92 & 1478 & 110 & 14.04 \\
\hline 46 & 82 & 1289 & 105 & 20.79 & 78 & 1475 & 31 & 14.03 \\
\hline 47 & 77 & 1288 & 15 & 20.71 & 68 & 1474 & 47 & 14.03 \\
\hline 48 & 63 & 1287 & 61 & 20.70 & 57 & 1466 & 41 & 14.02 \\
\hline 49 & 67 & 1287 & 23 & 20.67 & 39 & 1461 & 119 & 14.01 \\
\hline 50 & 101 & 1284 & 38 & 20.65 & 118 & 1460 & 78 & 14.01 \\
\hline 51 & 1 & 1281 & 9 & 20.64 & 81 & 1459 & 30 & 14.00 \\
\hline 52 & 40 & 1279 & 110 & 20.61 & 102 & 1450 & 68 & 13.99 \\
\hline 53 & 118 & 1275 & 116 & 20.56 & 75 & 1450 & 6 & 13.98 \\
\hline 54 & 41 & 1274 & 57 & 20.55 & 103 & 1449 & 48 & 13.97 \\
\hline 55 & 86 & 1272 & 84 & 20.55 & 16 & 1447 & 1 & 13.96 \\
\hline
\end{tabular}




\begin{tabular}{|c|c|c|c|c|c|c|c|c|}
\hline 1 & 104 & 1272 & 76 & 20.54 & 52 & 1446 & 9 & 13.95 \\
\hline 2 & 24 & 1267 & 35 & 20.52 & 101 & 1444 & 15 & 13.95 \\
\hline 3 & 80 & 1267 & 47 & 20.50 & 24 & 1435 & 80 & 13.94 \\
\hline 4 & 37 & 1266 & 104 & 20.49 & 116 & 1428 & 49 & 13.92 \\
\hline 5 & 18 & 1263 & 49 & 20.38 & 73 & 1426 & 76 & 13.92 \\
\hline 6 & 117 & 1257 & 41 & 20.37 & 48 & 1415 & 62 & 13.90 \\
\hline 7 & 56 & 1253 & 77 & 20.37 & 51 & 1409 & 38 & 13.87 \\
\hline 8 & 72 & 1253 & 80 & 20.35 & 67 & 1401 & 42 & 13.85 \\
\hline 9 & 38 & 1252 & 6 & 20.33 & 109 & 1397 & 116 & 13.85 \\
\hline 10 & 57 & 1251 & 19 & 20.33 & 85 & 1390 & 10 & 13.83 \\
\hline 11 & 15 & 1250 & 81 & 20.28 & 86 & 1388 & 117 & 13.80 \\
\hline 12 & 84 & 1249 & 32 & 20.27 & 70 & 1384 & 14 & 13.72 \\
\hline 13 & 64 & 1248 & 111 & 20.27 & 35 & 1383 & 100 & 13.68 \\
\hline 14 & 9 & 1246 & 65 & 20.22 & 47 & 1380 & 111 & 13.68 \\
\hline 15 & 51 & 1246 & 10 & 20.20 & 60 & 1374 & 65 & 13.67 \\
\hline 16 & 47 & 1244 & 1 & 20.15 & 55 & 1368 & 84 & 13.67 \\
\hline 17 & 52 & 1244 & 48 & 20.14 & 72 & 1361 & 32 & 13.67 \\
\hline 18 & 76 & 1242 & 118 & 20.12 & 113 & 1360 & 83 & 13.64 \\
\hline 19 & 102 & 1237 & 97 & 20.09 & 119 & 1360 & 19 & 13.60 \\
\hline 20 & 36 & 1234 & 109 & 20.08 & 36 & 1351 & 97 & 13.59 \\
\hline 21 & 70 & 1234 & 112 & 20.01 & 42 & 1345 & 51 & 13.59 \\
\hline 22 & 73 & 1228 & 51 & 19.96 & 77 & 1338 & 88 & 13.58 \\
\hline 23 & 14 & 1225 & 36 & 19.94 & 64 & 1331 & 35 & 13.57 \\
\hline 24 & 5 & 1222 & 64 & 19.92 & 107 & 1318 & 55 & 13.57 \\
\hline 25 & 112 & 1220 & 98 & 19.90 & 100 & 1312 & 77 & 13.54 \\
\hline 26 & 65 & 1218 & 117 & 19.89 & 37 & 1309 & 113 & 13.54 \\
\hline 27 & 75 & 1218 & 14 & 19.88 & 88 & 1308 & 118 & 13.53 \\
\hline 28 & 55 & 1218 & 55 & 19.85 & 10 & 1303 & 40 & 13.52 \\
\hline 29 & 110 & 1218 & 40 & 19.78 & 76 & 1299 & 98 & 13.50 \\
\hline 30 & 97 & 1212 & 70 & 19.78 & 18 & 1295 & 81 & 13.47 \\
\hline 31 & 10 & 1207 & 88 & 19.77 & 9 & 1277 & 36 & 13.43 \\
\hline 32 & 83 & 1205 & 5 & 19.73 & 38 & 1265 & 112 & 13.43 \\
\hline 33 & 48 & 1200 & 52 & 19.62 & 97 & 1245 & 70 & 13.40 \\
\hline 34 & 49 & 1200 & 100 & 19.51 & 49 & 1242 & 28 & 13.40 \\
\hline 35 & 111 & 1189 & 83 & 19.47 & 15 & 1240 & 71 & 13.40 \\
\hline 36 & 28 & 1180 & 71 & 19.43 & 110 & 1236 & 52 & 13.35 \\
\hline 37 & 88 & 1180 & 113 & 19.35 & 65 & 1226 & 64 & 13.34 \\
\hline 38 & 50 & 1175 & 34 & 19.32 & 112 & 1222 & 75 & 13.15 \\
\hline 39 & 107 & 1163 & 75 & 19.32 & 71 & 1222 & 34 & 13.13 \\
\hline 40 & 109 & 1162 & 73 & 19.22 & 84 & 1189 & 56 & 13.09 \\
\hline 41 & 66 & 1161 & 28 & 19.12 & 50 & 1163 & 107 & 12.99 \\
\hline 42 & 98 & 1155 & 66 & 19.05 & 111 & 1148 & 5 & 12.98 \\
\hline 43 & 113 & 1139 & 69 & 19.02 & 74 & 1135 & 73 & 12.96 \\
\hline 44 & 71 & 1138 & 107 & 19.02 & 5 & 1127 & 69 & 12.87 \\
\hline 45 & 100 & 1128 & 56 & 18.97 & 66 & 1126 & 66 & 12.69 \\
\hline 46 & 69 & 1103 & 50 & 18.80 & 98 & 1118 & 50 & 12.47 \\
\hline 47 & 44 & 1078 & 44 & 17.98 & 69 & 1062 & 44 & 12.06 \\
\hline 48 & 74 & 1021 & 74 & 17.51 & 44 & 1049 & 74 & 12.05 \\
\hline
\end{tabular}


Table 4. ANOVAs for means and standardized means of sites 1,2 , and 3 and for means and standardized means of sites $1,2,4$, and 5 .

Sites 1, 2, 3 means

Source of variation Degrees of freedom Mean square

Site $\quad 2 \quad 55,935,666$

Entry $\quad 119 \quad 32,063$

Site by entry $\quad 238 \quad 33,663$

Sites 1, 2, 3 standardized means

Source of variation Degrees of freedom Mean squares

Site $\quad 2 \quad 7,905$

Entry $119 \quad 2.71$

Site by entry $\quad 238 \quad 2.63$

Error infinity 1

Sites $1,2,4$, and 5 means

18 Source of variation Degrees of freedom Mean square

19 Site $35,59,627,911$

20 Entry $119 \quad 183,680$

21 Site by entry $357 \quad 114,771$

22

23 Sites 1, 2, 4, and 5 standardized means

24 Source of variation Degrees of freedom Mean square

25

26

Site

$3 \quad 18,907$

Entry -

Site by entry

1.54

Error

357 * $\quad 1.44$

infinity 1


1

2

3

4

6

7

8

9

10

11

12

13
Table 5. Individual ANOVAs for sites 1, 2, and 3.

\section{Site 1}

Source of variation $\quad$ Degrees of freedom Mean square

Entry $\quad 121 \quad 8,411$

$\begin{array}{lll}\mathrm{C} 1 & 1 & 12,953\end{array}$

$\begin{array}{lll}\mathrm{C} 2 & 1 & 48,712\end{array}$

$\begin{array}{lll}\mathrm{C} 3 & 1 & 42,867\end{array}$

$\begin{array}{lll}\mathrm{C} 4 & 1 & 22,613\end{array}$

$\begin{array}{lll}\text { C6 } & 1 & 31,220\end{array}$

$\begin{array}{lll}\mathrm{C} 8 & 1 & 77,300\end{array}$

$\begin{array}{llr}\text { R1 } & 1 & 28,678\end{array}$

$\begin{array}{lll}\text { R2 } & 1 & 12,832\end{array}$

$\begin{array}{lll}\text { R4 } & 1 & 4,993\end{array}$

$\begin{array}{lll}\text { R8 } & 1 & 20,170\end{array}$

R10 $\quad 1 \quad 15,068$

$\mathrm{C} 1 * \mathrm{R} 1 \cdots$

$\mathrm{C} 2 * \mathrm{R} 1 \quad 1 \quad 24,977$

C3*R1 1199

Residual (error) $\quad 44 \quad 3,449$

Site2

Source of variation Degrees of freedom Mean square

Entry $\quad 121 \quad 20,306$

$\begin{array}{lll}\mathrm{C} 1 & 1 & 37,044\end{array}$

$\begin{array}{lll}\mathrm{C} 4 & 1 & 56,069\end{array}$

$\begin{array}{lll}\mathrm{C} 10 & 1 & 24,484\end{array}$

$\begin{array}{lll}\mathrm{R} 2 & 1 & 108,367^{-}\end{array}$

$\begin{array}{lll}\mathrm{C} 1 * \mathrm{R} 1 & 1 & 218,001\end{array}$

$\begin{array}{lll}\mathrm{C} 1 * \mathrm{R} 3 & 1 & 30,779\end{array}$

$\begin{array}{lll}\mathrm{C} 2 * \mathrm{R} 2 & 1 & 17,944\end{array}$

$\mathrm{C} 2 * \mathrm{R} 4 \quad 1 \quad 46,852$

$\begin{array}{llr}\mathrm{C} 3 * \mathrm{R} 2 & 1 & 27,722\end{array}$

C3*R4 115

$\begin{array}{lll}\mathrm{C} 4 * \mathrm{R} 3 & 1 & 19,184\end{array}$

C4*R4 1133,627

$\begin{array}{lll}\text { Residual (error) } & 46 & 6,088\end{array}$

Site 3

Source of variation Degrees of freedom Mean square

Replicate

Entry

$11,149,108$

Block(replicate)

C1*block(replicate)

Residual (error)

1

119

212,224

$28 \quad 427,339$

$30 \quad 74,336$

$61 \quad 41,604$ 